\title{
The Study of displacement characteristics of Micro Defect Using vibration Infrared Thermography
}

\author{
by J.H. PARK*, H.S. PARK**, M.Y. Choi*, K.A. KWON*, W.J. Choi* and W.T. Kim*** \\ * Korea Research Institute of Standards and Science, Daejeon, South Korea, parkjh@kriss.re.kr \\ **Korea Research Institute of Smart Material and Structures System Association, Daejeon, South Korea, \\ m55nring@naver.com \\ ${ }^{\star * \star K o n g j u ~ N a t i o n a l ~ U n i v e r s i t y, ~ C h e o n a n, ~ S o u t h ~ K o r e a, ~ k w t @ k o n g j u . a c . k r ~}$
}

\section{Abstract (Arial, 9pt, bold)}

In recent years, Infrared thermography uses infrared energy radiated from any objects over absolute temperature, and the range of its application has been constantly broadened. The infrared thermography is an effective technique to detect cracks detection by using ultrasound infrared thermography for non-destructive testing method. This study sought to enhance the rate of defects detection by generating the maximum displacement with the use of ultrasonic excitation based on the study of multi-excitation characteristics. if the multi-excitation that can apply the excitation to several exciters at the same time in specimens of large-sized objects is utilized.

\section{Introduction}

In recent years, a thermal behavior measuring technology using infrared rays has been utilized in various fields. The methods of detecting defects by using infrared thermography are largely divided into passive methods and active methods. As for the excitation energy, various energies such as light source, ultrasound, vibration, micro-wave, induced current, hot air and cold air can be used in an active testing method. Of all the experimental parameters for the ultrasound infrared thermography, the excitation energy is the biggest variable. In order to solve this problem, this study sought to enhance the rate of defects detection by generating the maximum displacement with the use of ultrasonic excitation based on the study of multi-excitation characteristics[2].

\section{Experimental Materials and Apparatus}

\subsection{Experimental Materials}

With the use of fatigue crack specimens, an experiment was performed to identify the size of a defect and the position of heat generation. The specimen used in the experiment was made of carbon steel and produced through a fatigue test after the generation of notches, and its shape is shown in Fig. 1 . The thickness of the test specimens is 20 $\mathrm{mm}$

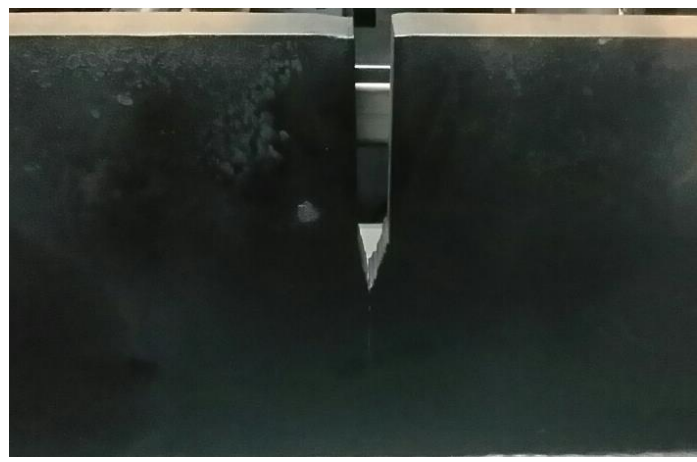

Fig. 1. Fatigue crack specimen

\subsection{Experimental Apparatus}

For generation of ultrasound, a piezoelectric element was used, and two exciters that generate a natural frequency of $19.8 \mathrm{kHz}$ were also used. At the moment when ultrasound vibration pulse is impressed, the ultrasound 
infrared thermography equipment consists of ultrasound excitement equipment and an infrared thermography camera, and in order to minimize the heat exchange between test specimen and outer heat source during the test, experimental equipment is installed inside the insulation chamber as shown in Fig. 2. The infrared excitement equipment has 400 Watt of output and $19.8 \mathrm{kHz}$ of frequency, while Silver $480 \mathrm{~m}$ Model (NEDT: $25 \mathrm{mK}$ ) made in French Cedip was used for the infrared camera. An optical fiber displacement sensor was used to measure the displacement of the specimen to be subjected to the excitation.

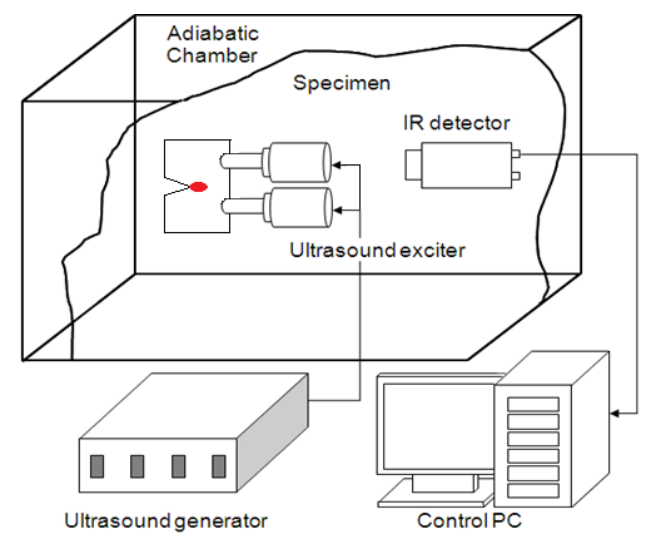

Fig. 2. A config of the experimental setup

\section{Experimental Result}

In the experiment, the defects detection and displacement measurements were performed by locating exciters at the same position on both sides, $150 \mathrm{~mm}$ away from each other around the defect and then operating one exciter and two exciter at the same time. For the displacement measurements, philtec-62 C fiber optic displacement measuring device was used. The resolution of the displacement measuring device is $0.1 \mu \mathrm{m}$. A force of $10 \mathrm{kgf}$ was applied so that the excitation horn can give the same force to specimens for excitation. Fig.3 (a) shows the thermal image of the defect that occurs in the specimen when a single excitation was performed with one exciter. When one exciter was used, the displacement increased at an average of less than $2.5 \mu \mathrm{m}$. Based on these results, it was found that when two exciters with the same natural frequency were under multi-excitation, a more precise infrared themography defect detection image was obtained as in Fig. 3 (b) than as in Fig. 3 (a). The results of the displacement that occurs in the specimen under multi-excitation revealed that the amount of displacement increases at a rate of less than $6 \mu \mathrm{m}$ on average as shown in Fig. 4.[3] However, it was confirmed that the effects occurred due to the displacement amplification only when two horns operated at the same time during the excitation, and each excitation cycle which operates differently had an effect on the detection of defects. If the excitation cycle varies at the time of excitation with the excitation cycle of a sine wave, the energy cannot be maximized due to a failure to operate the vibration energy at a constant level. This makes it difficult to perform the frequency synchronization of lock-in test, and thus poses a difficulty in detecting the defects.

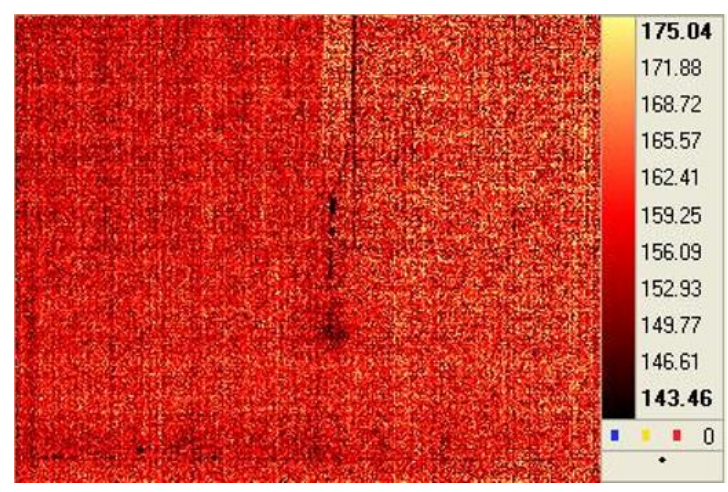

(a) single excitation

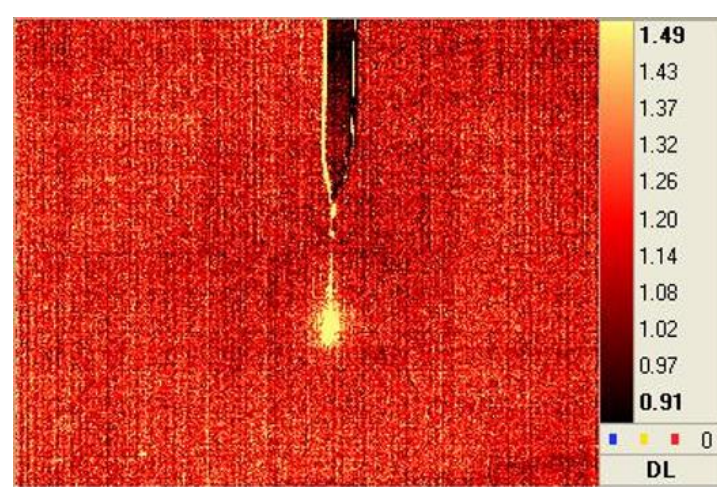

(b) Multi excitation

Fig. 3. Ultrasound Infrared thermography of single excitation and multi excitation. 


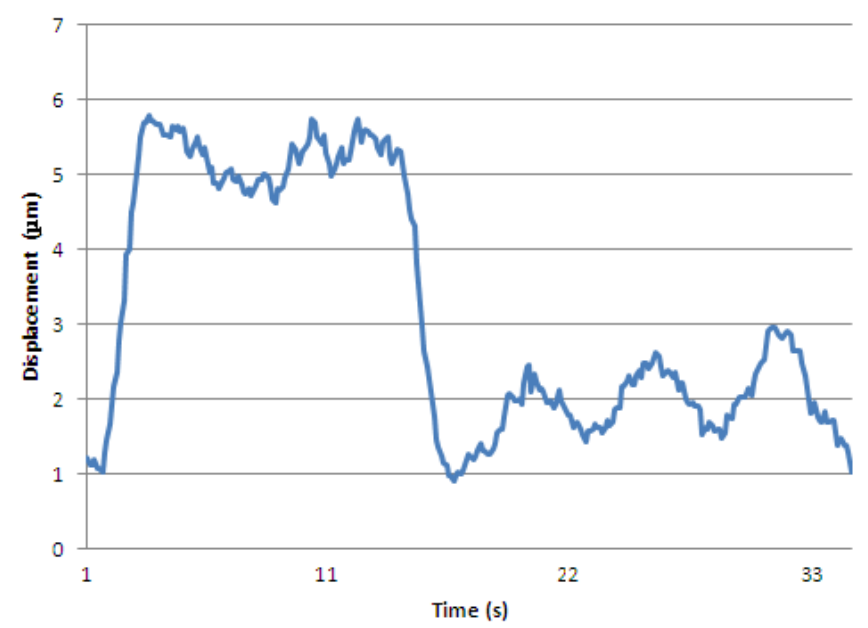

Fig. 4. Displacement measure of single excitation and multi excitation

\section{Conclusion}

Among various testing techniques for effective use of the ultrasound infrared thermograhy, a method for amplifying the displacement of the test specimen, while maintaining the natural frequency by using multi-excitation was used for the defects detection. Through this method, it was confirmed that if two exciters with the same frequency are used, the amplification of displacement occurs, and the detection of defects is effective in comparison with the case of using one exciter. In the future, if the multi-excitation that can apply the excitation to several exciters at the same time in specimens of large-sized objects is utilized, the method is expected to serve as a more effective tool for detecting the defects in thick metal and composite materials.

\section{ACKNOWLEDGEMENT}

This work was supported by the National Research Foundation of Korea (NRF) grant funded by the Korea government (MSIP) (NRF-2013M2A 2A9043706), (NRF-2015R1A2A2A01005426)

\section{REFERENCES}

[1] R. B. Mognogna, R. E. Green Jr., J. C. Duke Jr., E. G. Henneke II and K. L. Reifsnider, "Thermographic investigation of high-power ultrasonic heating in materials," Ultrasonics, Vol. 19, pp. 159-163 (1981)

[2] Myunghwan Kang, Jongju Yi, Seungjin Han, Sooryong Bae and Woojin Jung, "Analysis of the Acoustic Radiation Efficiency on Multi-excitation System with Different Phase" Transactions of the Korean society for noise and vibration engineering, 24 no.12, 2014 pp.992-998

[3] H. S. Park, M. Y. Choi, J. H. Park, K. A. Kwon, W. J. Choi, S. U. Kim , "Study on the micro crack detection in defect zone by using multi ultrasound thermography" autumn Conference 2015, KSNT 